\title{
Development of New Products by ISM
}

\author{
Shih-Wen Hsiao \\ Department of Industrial Design, \\ National Cheng Kung University, \\ Tainan, Taiwan 70101, ROC \\ swhsiao@mail.ncku.edu.tw
}

\author{
Chi-Hung Lo \\ Department of Industrial Design, \\ Tunghai University, \\ Taichung, Taiwan, $R O C$ \\ chlo@thu.edu.tw
}

\author{
Hsin-Hung Lin \\ Department of Industrial Design, \\ National Cheng Kung University, \\ Tainan, Taiwan 70101, ROC \\ a123lin0@gmail.com
}

\begin{abstract}
The key to success in the development of a new product is to determine the relationships between components of the product. Therefore, an effective method which clarifies the relationship between components is an important factor for the success in product developments. To resolve this issue, the method of interpretive structural model (ISM), , The component framework with the best weight can been obtained, and can be applied to determine the relationships between product components during the process of new product development. By utilizing an effective and systematic grouping method, and by the integration with the standpoint of the development and manufacturing of new products, there are four product series established from the results of the case study. And the capability of manufacturing and assembling common components and individual components between product series of both sides can be established. New manufacturing and assembling capabilities can be expected to be developed and the cost can thus be reduced. The target of providing product multiplicity, reducing component counts for the purpose of the development and management of mass-produced products can be achieved, and the product development time can be effectively reduced.
\end{abstract}

Keywords-c modular design; product family; interpretive structural model; product design

\section{INTRODUCTION}

The purpose of this study is to enhance the production and manufacturing efficiency. By proposing the concept of concurrent engineering, this study aims at assisting designers in considering the manufacturing problem during design stages, and reducing the occurrence of potential manufacturing problems to the best of their abilities.
The processes of the development, manufacturing and assembly of new products are the critical elements of a synchronous production manufacturing. It is important to develop an engineering method, which satisfies various customer demands on a highly competitive market, for the product manufacturing and assembly processes. Hsiao et al. (2013) [1] proposed an approach of product segmentation, which can reduce costs and enhance product diversity, in the product development process to satisfy the modularization of various customer demands. [2] The technique of ISM have been applied to the hierarchical structures of visualized products, with which the ingredients interact with each other. The priority and related structural charts employed by such a kind of design method take aim at different market demands with the goal to develop solution packages during the process of product development [3]. Therefore, enhancing assembling efficiency is vital to the enhancement of product competitiveness. In most cases, the most critical element during the design process of product development is cost, while the influence of the manufacturing process on the total cost is rather smaller. Any changes in the product structure, appearance, or other related designs will lead to additional increase in the production cost. The concept of concurrent 
engineering can be integrated into design stages to solve this problem to the core. The additional costs due to unnecessary design changes can be prominently reduced, and consequently the manufacturing efficiency can be increased.

\section{OUTLINE OF THE DEVELOPMENT PROCESS FOR THE RESEARCH MODEL}

In this study, products have been decomposed to determine the modular relationship between them and the relation matrix of modularization between markets can be acquired. This relation matrix was further utilized for determining the optimal product module and the flowchart of this research process is Firstly, the modularization analysis of components can be conducted by ISM, and the methods of DEI and RAC. The implementation procedure is as follows:

(1) Construct the relation matrix.

(2) Generate the reachability matrix.

(3) Export the matrix.

(4) A rearranged matrix can be formed by the relation matrix and the reachability matrix that have been built.

(5) Illustrate the hierarchical relationships between element modules.

(6) Distribute the relationships between modules.

(7) Calculate RAC between parts

\section{THE MODULE CONCEPT}

The a in this study for the analysis of each task. The methods that are going to be used in this study are explained respectively as follows.

\section{A. Basic concepts and development processes of ISM}

Based on the relativity between elements in a system, matrix operations have been used for the assessment of customer demands. The resulting hierarchical relationships in the figures obtained can be used to constitute strategies and solutions to the problems [4]. ISM, which was first introduced by Warfield, can be used to calculate and decompose problems [1]. It has already been used in various research domains, and can reduce the interacting logics and orientations in a complicated system to its maximum extent [2]. Hsiao and Liu [3] utilized the improved ISM to establish a stratified and interlacing component structure, which can help designers confirm the commonality, diversity, and design emphasis of components [5]. Therefore, this approach assists designers in developing a product series [8]. The procedure of ISM is described as follows.

\section{Step 1: Construct a relation matrix}

Conduct logical operations and an analysis of the hierarchical structures: Arrange the elements (entries) of the problem in a form of matrix to select and sample the structure elements and compare the relations between the elements. To compare the relations, a directional relation matrix [A] is formed by using the relation $\left(a_{i j}\right)$ between one element and another. The four major principles are as follows:

(1) For the constituent element $\mathrm{a}_{\mathrm{ij}}$, if $\mathrm{i}$ has an impact on $\mathrm{j}$, $\mathrm{a}_{\mathrm{ij}}=1$; if not, $\mathrm{a}_{\mathrm{ij}}=0$.

(2) For the constituent element $a_{j i}$ in the reverse direction, if $\mathrm{j}$ has an impact on $\mathrm{i}, \mathrm{a}_{\mathrm{ji}}=1$; if not, $\mathrm{a}_{\mathrm{ji}}=0$.

(3) If the two elements do not affect each other, $a_{\mathrm{ij}}=0$ and $\mathrm{a}_{\mathrm{ji}}=0$.

(4) If the two elements affect each other, $\mathrm{a}_{\mathrm{ij}}=1$ and $\mathrm{a}_{\mathrm{ji}}=1$.

Fig.1(a) represents the incidence matrix of an example system which contains seven components, and displays the incidence relationships between components. For example, the second row of the matrix indicates that component 3 directly affects components 1,5 , and 7 .

\section{Step 2: Generate a reachability matrix}

The reachability matrix $[R]$ is deducted from the incidence matrix [A] if a Boolean n-multiple product of $[\mathrm{A}]+[\mathrm{I}]$ uniquely converges to $\mathrm{R}$ for all integers $n>n_{0}$, where $n_{0}$ is an appropriate positive integer, [I] is a Boolean unity matrix, and + is addition in Boolean sense. Matrix [R] represents all direct and indirect connections 
between components. Relation transitivity is a basic assumption in ISM. Fig. 1(b) represents the reachability matrix $[R]$ derived from matrix $[A]$, in which an entry $r_{i j}=1$ if component $j$ is reachable by $i$, although the path length may be one or more. "Reachability": In graph theory, reachability is the ability to get from one vertex in a directed graph to some other vertex. It is sufficient to find the connected components in the graph.

\section{Step 3: Generate a pairwise output matrix}

According to the result of the reachability matrix, select elements with aij=aji $=1$; that is, the elements that affect each other (aij=1and aji=1). A technique for cluster retrieval is inserted in the ISM process to identify components that influence one another and form a loop. Similar algorithm has been used in the graph theory. The reachability matrix $[R]$ multiplies the transposed matrix of $[\mathrm{R}]$, say $[\mathrm{R}]^{\mathrm{T}}$; thus in $[\mathrm{R}] \cdot[\mathrm{R}]^{\mathrm{T}}$, components $i$ and $j$ mutually interact if $r_{i j} r_{j i}=1$. Fig. 1(c) displays the output matrix of $[R] \cdot[R]^{T}$, in which clusters of components can be identified easily by rearranging component order.

\section{Step 4: Generate a rearranged matrix}

Clusterize the elements that affect one another in the output matrix of the reachability matrix. Fig. 1(d) reveals four clusters in the system, namely $\{1\},\{2,6\},\{3,5,7\}$, and $\{4\}$. Trace back to the element correlations in the reachability matrix to generate a rearranged matrix. The order of the reachability matrix $[R]$ is rearranged (as shown in Fig. 1(e)), and the clustered components are integrated and treated as a single entity.

\section{Step 5: Illustrate the element hierarchical relationships}

The hierarchy graph then is obtained by identifying a set of components in matrix $\mathrm{R}$ that cannot reach or be reached by other components outside the set itself, removing the set from the original matrix $[R]$, and then repeating this process for remaining matrix until a unique set of nodes that no other nodes can reach is obtained. For example, in Fig. 1(e), $c_{1}$ is first identified as an "exit", that is, the lowest level of the hierarchy, since it cannot reach to other components; meanwhile, $\left\{c_{2}, c_{6}\right\}$ and $c_{4}$ were separated as "entrances", because they cannot be reached by other nodes. In this example, three levels of nodes were obtained (illustrated in Fig. 1(f)). The oriented links then connected the nodes from source to sink one (for example, from $\mathrm{c}_{4}$ to $\mathrm{c}_{5}$ ) based on the incidence matrix. Notably, the rounded rectangles in Fig. 1(f) indicate clusters retrieved in procedure 3 , in which the information flow forms a loop. At this step, element hierarchical relationships are illustrated according to the rearranged matrix, providing decision makers with the procedures and hierarchical structure in the deconstruction of a problem.

\section{Step 6: Draw the D+R_D-R element distribution graph}

According to the reachability matrix, add up the scores of the elements in each row to generate $\mathrm{D}$ and the elements in each column to generate R. Calculate the values of $D+R$ and $D-R$ to generate a reachability matrix determinant (as shown in Fig. 2). Demonstrate D+R_D-R on a binary scale to interpret both the problem and the target areas. It can also be employed to analyze independent elements, correlated elements, dominant elements and dominated elements, which are demonstrated in Fig. 3.

For designers, ISM has the capability of clarifying the interactions between element attributes. It is no longer required to just depend on own experiences or instinctual manners to process the data, rather it is required to find the critical attributes among complex and multiple relations and try to develop upon them, and transform originally individual data into valuable information and finally strategic methods for design and planning are utilized [7]. Because of this, ISM 
has been introduced into this study to assist designers in transforming demands into real product characteristics [6].
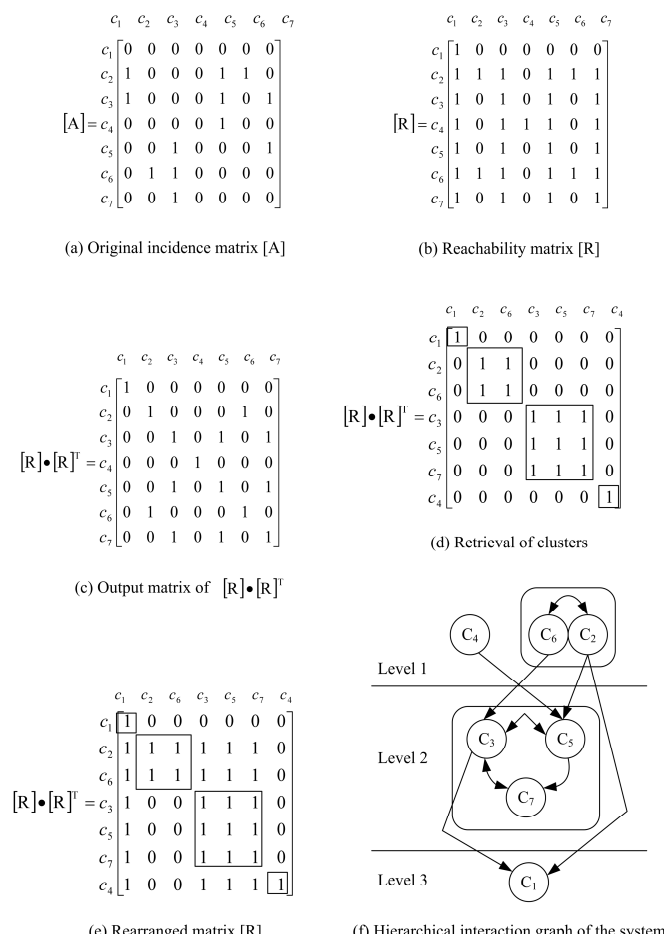

(f) Hierarchical interaction graph of the system

FIG.1. Step-by-step process of ISM.

\begin{tabular}{rllllllllrr}
\hline$a_{i j}$ & $C_{1}$ & $C_{2}$ & $C_{3}$ & $C_{4}$ & $C_{5}$ & $C_{6}$ & $C_{7}$ & $\mathrm{D}$ & $\mathrm{D}+\mathrm{R}$ & $\mathrm{D}-\mathrm{R}$ \\
\hline$C_{1}$ & 1 & 0 & 0 & 0 & 0 & 0 & 0 & 1 & 8 & -6 \\
$C_{2}$ & 1 & 1 & 1 & 0 & 1 & 1 & 1 & 6 & 8 & 4 \\
$C_{3}$ & 1 & 0 & 1 & 0 & 1 & 0 & 1 & 4 & 10 & -2 \\
$C_{4}$ & 1 & 0 & 1 & 1 & 1 & 0 & 1 & 5 & 6 & 4 \\
$C_{5}$ & 1 & 0 & 1 & 0 & 1 & 0 & 1 & 4 & 10 & -2 \\
$C_{6}$ & 1 & 1 & 1 & 0 & 1 & 1 & 1 & 6 & 8 & 4 \\
$C_{7}$ & 1 & 0 & 1 & 0 & 1 & 0 & 1 & 4 & 10 & -2 \\
$\mathrm{R}$ & 7 & 2 & 6 & 1 & 6 & 2 & 6 & & & \\
\hline
\end{tabular}

FIG. 2. Reachability matrix determinant.

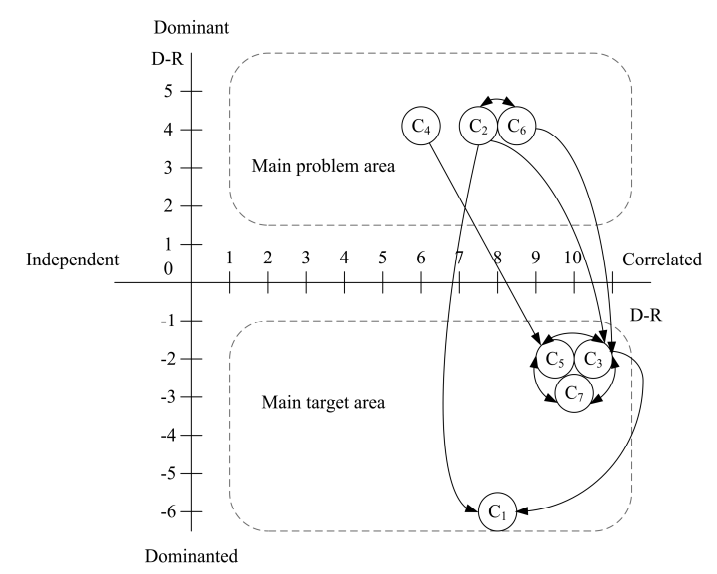

FIG. 3. D+R_D-R Element Distribution Graph.

\section{B. Correlation matrices between parts}

The assembly sequence is a critical factor for the production of products. The determination of assembly sequence is typically based on subjective experiences of assembly design. The construction of network figures after assembly can be used for the investigation of correlations between parts. For the engineering characteristics between product replacements and parts, the time required during the process of assembling parts can be considered as one of the major indicators.

\section{Disassembly Effort Index (DEI)}

Das et al. [1,11,12] pairwise RAC relationships between parts are scored.

The main purposes for using the above three pieces of engineering information to evaluate assembly relationships are as follows:

(1) Contact method: Contact methods can be roughly divided into four categories: moveable and can be disassembled, fixed and can be disassembled, movable and cannot be disassembled, and fixed and cannot be disassembled.

(2) Tool: Tools help to complete an assembly action, so it is very important to choose the right one. Parts must be assembled under certain circumstances; likewise, there is a specific standard for using tools. Each kind of assembly and disassembly has its suitable tool(s).

(3) Assembly direction: It is inevitable to consider assembly directions when parts are assembled or disassembled. Directions can be multi-dimensional. Parts can be assembled at different angles and in different directions. Therefore, considering directions is one of the essential elements in the planning of product assembly and disassembly. Moreover, an improved minimal spanning tree is employed to seek network relationships.

a. All assembly relationships can be reversed in direction; for example, $\{\mathrm{C} 1 \rightarrow \mathrm{C} 2\}$ or $\{\mathrm{C} 2 \rightarrow \mathrm{C} 1\}$. 
b. RAC weight values are determined by obtaining distance relationships by means of an attribute matrix.

c. An improved minimal spanning tree is employed to obtain assembly network relationships, and repeatedly selected assembly relationships are removed. For example, $\{\mathrm{C} 1 \rightarrow \mathrm{C} 2\}$ or $\{\mathrm{C} 2 \rightarrow \mathrm{C} 1\}$ (that is, when $\mathrm{d}_{\mathrm{ij}}=\mathrm{d}_{\mathrm{ji}}$, only assembly relationships that have appeared before are recorded.)

\section{Calculation of RAC weights}

Before starting the substitutions in the algorithm, the above-mentioned engineering information must be combined in order to facilitate the operation of the algorithm. This study attempts to use the concept of relative distance in a vector space to construct a relationship distance matrix between parts. Three pieces of the engineering information are treated as three dimensions in a vector space, which are integrated through distance. The corresponding relationships of engineering properties are considered from the standpoint of distance, and each assembly relationship between parts is a node in the space, corresponding to a certain engineering attribute. The corresponding relation matrix is substituted into a distance formula (Eq. 1) to determine the distance between each node and the origin, indicating the differences in assembly relationships $[9,10]$.

$d_{i j}=\sqrt{\left(x_{i}-x_{0}\right)^{2}+\left(y_{i}-y_{0}\right)^{2}+\left(z_{i}-z_{0}\right)^{2}}$

$\mathrm{d}_{i j}$ : The distance between a parts relationship and the origin

$\mathrm{x}_{i}$ : Corresponding scores of "type" attribute

$\mathrm{y}_{i}$ : Corresponding scores of "tool" attribute

$\mathrm{z}_{i}$ : Corresponding scores of "access" attribute

$\left(\mathrm{x}_{0}, \mathrm{y}_{0}, \mathrm{z}_{0}\right)$ : Coordinates of the origin

Origin: the rightmost assembly relationship value corresponding to each attribute criterion

The RAC degrees of matched-pair relationships are based on the assessment table (as shown in Fig. 4), and calculations have been conducted to obtain the distance between every node and the origin, which indicates the difference in assembly relationships. The results can be substituted into the distance equation by the matrix of the corresponding relationship (Eq. 2).

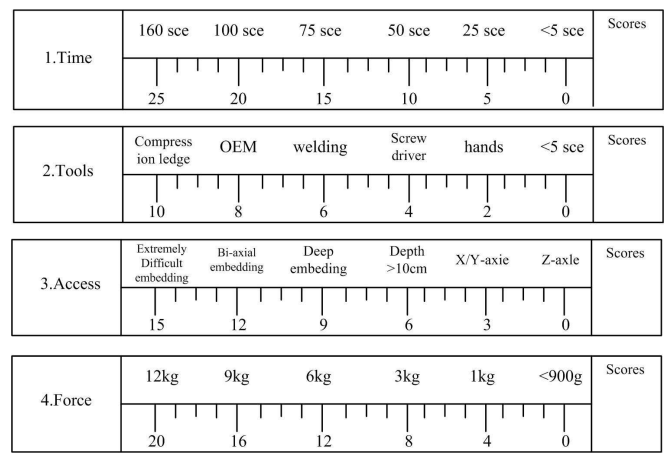

FIG. 4. Modified Disassembly Effort Index.

$$
R A C=\frac{1}{\sqrt{(T i)^{2}+(T o)^{2}+(A c)^{2}+(F o)^{2}}}
$$

\section{RAC: Distance between parts}

Ti: Indicator value between parts according to the "time" criterion

To: Indicator value between parts according to the "tools" criterion

Ac: Indicator value between parts according to the "access" criterion

Fo: Indicator value between parts according to the "force" criterion

The main(qalculation process is divided into two steps: Step 1: Calculate the RAC distance values in the modules.

Step 2: Calculate the RAC distance values outside the modules.

\section{APPLICATION OF CASE STUDY AND CASE VERIFICATION}

To verify the feasibility of this study, a series of studies has been proposed and the operating process is explained in details as follows.

The design of a radiant electric heater has been selected as the case study. Samples of radiant electric heater which are available at the moment have been collected to cross-check the conventional parts, i.e., the essential accessories of an electric 
heater. The three-dimensional model and the exploded view of parts are as shown in Fig. 5.

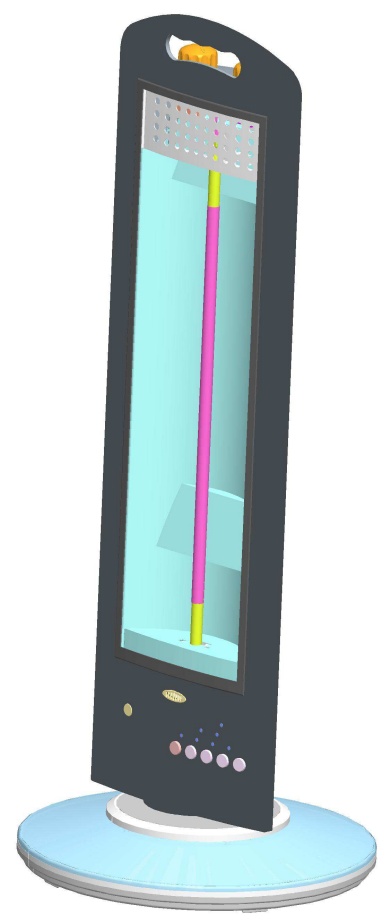

(a) Three-dimensional model

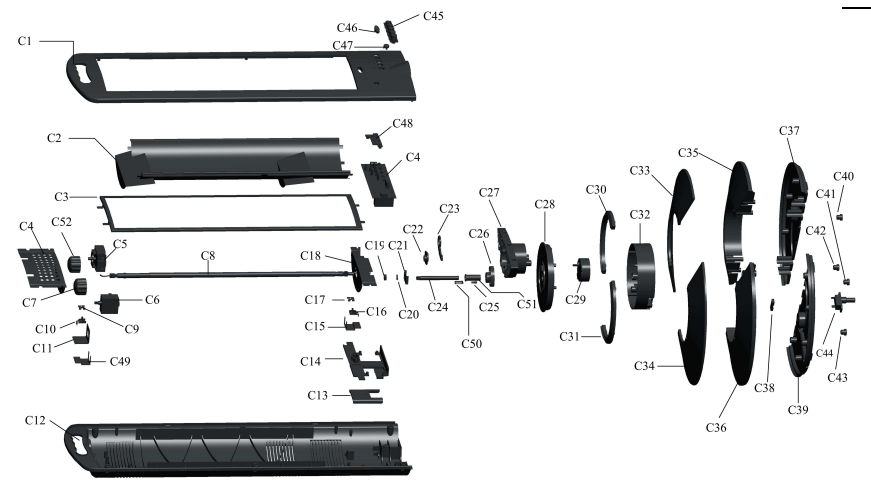

(b) Exploded view of parts

FIG. 5. Three-dimensional model and the exploded view.

\section{A. ISM calculations}

The relations between parts of a radiant electric heater can be established as a relation matrix by means of ISM. The $52 * 52$ matrix can be further processed by the ISM technique, which has been described, the D+R_D-R values of the element distribution can also be obtained as shown in Table 1
TABLE 1. D+R_D-R values of the element distribution by ISM.

\begin{tabular}{cccccccccccccccccc}
\hline & $\mathrm{C1}$ & $\mathrm{C} 2$ & $\mathrm{C} 3$ & $\mathrm{C} 4$ & $\mathrm{C} 5$ & $\mathrm{C} 6$ & $\mathrm{C} 7$ & $\mathrm{C} 8$ & $\mathrm{C} 9$ & $\mathrm{C10}$ & $\mathrm{C11}$ & $\mathrm{C12}$ & $\mathrm{C13}$ & $\mathrm{C14}$ & $\mathrm{C15}$ & $\mathrm{C16}$ & $\mathrm{C17}$ \\
\hline $\mathrm{D}$ & 30 & 38 & 31 & 30 & 38 & 40 & 40 & 38 & 40 & 40 & $\mathbf{1}$ & $\mathbf{1}$ & $\mathbf{1}$ & $\mathbf{1}$ & $\mathbf{1}$ & 40 & 39 \\
\hline $\mathrm{R}$ & 40 & $\mathbf{1 2}$ & $\mathbf{1}$ & 40 & $\mathbf{1 2}$ & 2 & 2 & $\mathbf{1 2}$ & 2 & 2 & $\mathbf{1 3}$ & $4 \mathbf{1}$ & $\mathbf{1}$ & $4 \mathbf{1}$ & $\mathbf{1 3}$ & $\mathbf{1}$ & 2 \\
\hline $\mathrm{D}+\mathrm{R}$ & 70 & 50 & 32 & 70 & 50 & 42 & 42 & 50 & 42 & 42 & $\mathbf{1 4}$ & 42 & 2 & 42 & $\mathbf{1 4}$ & $4 \mathbf{1}$ & $4 \mathbf{1}$ \\
\hline $\mathrm{D}-\mathrm{R}$ & $-\mathbf{1 0}$ & 26 & 30 & $\mathbf{- 1 0}$ & 26 & 38 & 38 & 26 & 38 & 38 & $\mathbf{- 1 2}$ & -40 & 0 & -40 & $\mathbf{- 1 2}$ & 39 & 37 \\
\hline
\end{tabular}

C18 C19 C20 C21 C22 C23 C24 C25 C26 C27 C28 C29 $\mathrm{C} 30$ C31 C32 C33 C34

\begin{tabular}{cccccccccccccccccc}
$\mathrm{D}$ & 38 & 30 & 30 & 30 & 33 & 31 & 30 & 30 & 30 & 30 & 30 & 33 & 30 & 30 & 30 & 30 & 30 \\
\hline $\mathrm{R}$ & $\mathbf{1 2}$ & 40 & 40 & 40 & 2 & 3 & 40 & 40 & 40 & 40 & 40 & 2 & 40 & 40 & 40 & 40 & 40 \\
\hline $\mathrm{D}+\mathrm{R}$ & 50 & 70 & 70 & 70 & 35 & 34 & 70 & 70 & 70 & 70 & 70 & 35 & 70 & 70 & 70 & 70 & 70 \\
\hline $\mathrm{D}-\mathrm{R}$ & 26 & $\mathbf{- 1 0}$ & $\mathbf{- 1 0}$ & $\mathbf{- 1 0}$ & 31 & 28 & $\mathbf{- 1 0}$ & $\mathbf{- 1 0}$ & $\mathbf{- 1 0}$ & $\mathbf{- 1 0}$ & $\mathbf{- 1 0}$ & 31 & $\mathbf{- 1 0}$ & $\mathbf{- 1 0}$ & $\mathbf{- 1 0}$ & $\mathbf{- 1 0}$ & $\mathbf{- 1 0}$
\end{tabular}

\begin{tabular}{lllllllllllllllllll}
\hline C36 & C37 & C38 & C39 & C40 & C41 & C42 & C43 & C44 & C45 & C46 & C47 & C48 & C49 & C50 & C51 & C52
\end{tabular}

\begin{tabular}{ccccccccccccccccc}
\hline 30 & 6 & 31 & 6 & 6 & 7 & 6 & 6 & 6 & 30 & 30 & 30 & 30 & 38 & 30 & 31 & 38 \\
\hline 40 & 47 & $\mathbf{1}$ & 47 & 47 & $\mathbf{1}$ & 47 & 47 & 47 & 40 & 40 & 40 & 40 & $\mathbf{1 2}$ & 40 & $\mathbf{1}$ & $\mathbf{1 2}$ \\
\hline 70 & 53 & 32 & 53 & 53 & 8 & 53 & 53 & 53 & 70 & 70 & 70 & 70 & 50 & 70 & 32 & 50 \\
\hline $\mathbf{- 1 0}$ & $-\mathbf{4 1}$ & 30 & $-4 \mathbf{1}$ & -41 & 6 & -41 & -41 & -41 & $-\mathbf{1 0}$ & $\mathbf{- 1 0}$ & $\mathbf{- 1 0}$ & $\mathbf{- 1 0}$ & 26 & $\mathbf{- 1 0}$ & 30 & 26
\end{tabular}

\section{B. Establishment of modular clusters}

Four modularized clusters can be generated from the rearranged matrix as shown in Fig. 5. These four modularized clusters are listed respectively as follows:

Cluster 1: $\mathrm{M} 1=\{\mathrm{C} 2, \mathrm{C} 5, \mathrm{C} 8, \mathrm{C} 52, \mathrm{C} 18\}$. Cluster 1 contains seven parts. It is the module with the most parts in this case.

Cluster 2: M2 $=\{\mathrm{C} 3, \mathrm{C} 6, \mathrm{C} 7, \mathrm{C} 9, \mathrm{C} 10, \mathrm{C} 11, \mathrm{C} 12 \mathrm{C} 13, \mathrm{C} 14$, $\mathrm{C} 15, \mathrm{C} 16, \mathrm{C} 17\}$. Cluster 12 contains 3 parts. It is the module with the fewest parts in this case.

Cluster 3: M3 $=\{\mathrm{C} 1, \mathrm{C} 4, \mathrm{C} 19, \mathrm{C} 20, \mathrm{C} 21, \mathrm{C} 24, \mathrm{C} 25, \mathrm{C} 26$, C27, C28, C30, C31, C32, C33, C34, C35, C36, C45, C46, $\mathrm{C} 47, \mathrm{C} 48, \mathrm{C} 50\}$.

Cluster 4: M4 $=\{\mathrm{C} 22, \mathrm{C} 29, \mathrm{C} 23, \mathrm{C} 37, \mathrm{C} 39, \mathrm{C} 40$, C42,C43,C44,C51,C38,C41\}.

\section{Calculation of RAC weights}

RAC and distances. The resulting values of RAC and distances of modules of an electric heater are shown in Fig.6. An RAC value which is closer to " 0 " indicates a stronger interaction, otherwise a larger value indicates an interaction which is more relaxed. 


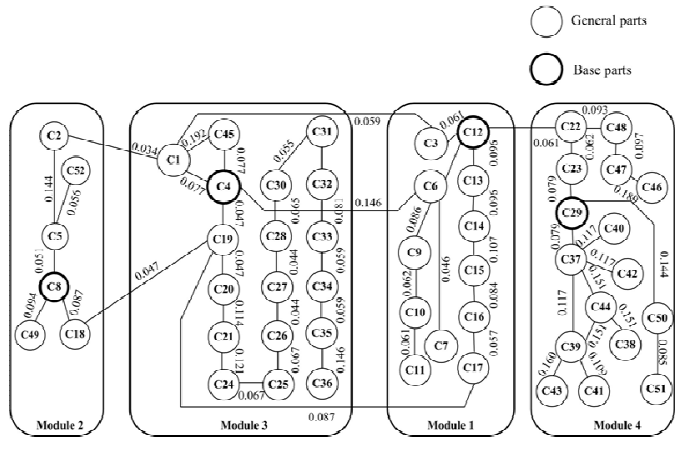

FIG. 6. Resulting values of RAC of radiant electric heater modules.

\section{Acquisition of element weights}

This result can serve as a reference for the development of a plurality of product series during the modularized design process. Based on the market segments proposed in this study, it is recommended to design four product lines, which correspond respectively to the demands of different segmented markets and supply manufacturing benefits related to costs and modularized products. Three types of conditions have been studied, and the results forms Fig. 7 satisfying the two requirements of modularization and market segmentation.

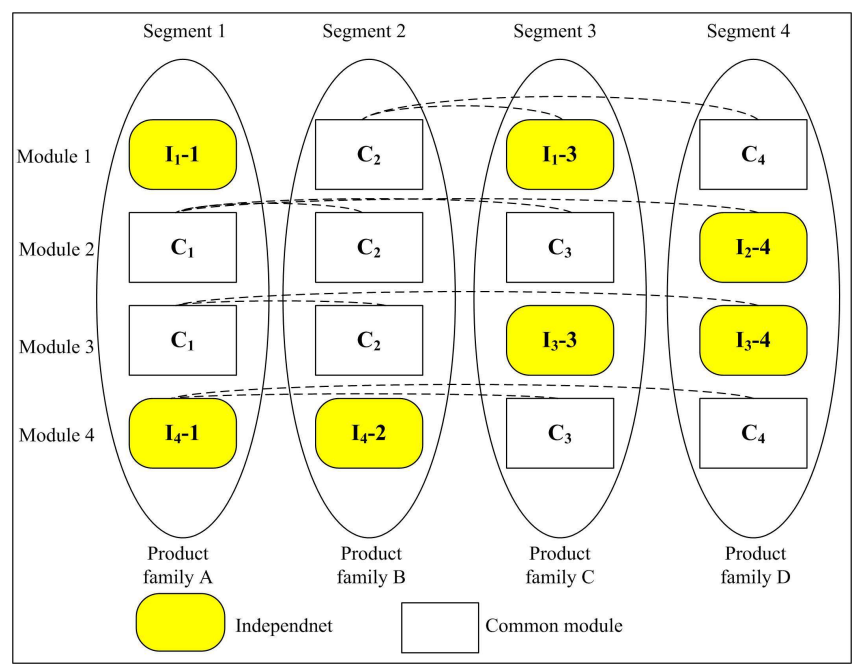

FIG. 7. Compositions of common modules and independent modules.

\section{CONCLUSIONS AND SUGGESTIONS}

A modularized and systematic structure has been established in this study by a case study on the product design of electric heaters. An applicable method of modularization and clusterization by ISM has been determined, the module presentation with the optimal weights can be obtained. Four product series have been established according to the results of the case study, and the assembly performance of the common and individual components between product series can be determined. The proposed method can be applied to the beginning stage of new product development, and more particularly to the design process of different product series. Except for developing suitable product lines, it can also be applied to the establishment of product modules for every segmented market.

\section{Acknowledgement}

The authors would like to thank the Ministry of Science and Technology of Taiwan, ROC for the financial support under grant number MOST 105-2221-E029-011.

\section{References}

[1] Shih-Wen Hsiao, Ya-Chuan Ko, Chi-Hung Lo, Shih-Ho Chen, (2013).An ISM DEI and ANP based approach for product family development. Advanced Engineering Informatics. 27 131-148.

[2] Shih-Wen Hsiao, Elim Liu(2005). A structural component-based approach for designing product family. Computers in Industry. 56 13-28

[3] T.H. Eng, Z.K. Ling, W. Olson, C. McLean, (1999) Feature-based assembly

[4] Ali Diabat,Kannan Govindan (2011).An analysis of the drivers affecting the implementation of green supply chain management. Resources, Conservation and Recycling 55 659-667.

[5] V. Ravi, Ravi Shankar(2005).Analysis of interactions among the barriers of reverse logistics. Technological Forecasting \& Social Change 72 1011-1029.

[6] Ashish Agarwal, Ravi Shankar, M.K. Tiwari (2007).Modeling agility of supply chain. Industrial Marketing Management 36 443-457.

[7] Wei-Ming Wang, Amy H.I. Lee, Li-Pei Peng, Zih-Ling Wu(2013) . An integrated decision making model for district revitalization and regeneration project selection. Decision Support Systems 54 1092-1103.

[8] Víctor. Bañulsa, Murray Turoff(2011).Scenario construction via Delphi and cross-impact analysis. Technological Forecasting \& Social Change 78 1579-1602.

[9] T. Kitamura, A computer-aided approach to the structural analysis and modification of a large circulatory system model, IEEE Trans. Biomed. Eng. 46 (1999) 485-493.

[10] H.D. Sharma, A.D. Gupta, Sushil, The objectives of waste management in India: a futures inquiry, Technol. Forecast. Soc. Chang. 48 (1995) 285-309.

[11] S.K. Das, P. Yedlarajiah, R. Narendra, An approach for estimating the end-of-life product disassembly effort and cost, Int. J. Prod. Res. 38(3) (2000) 657-673.

[12] Amy H.I. Lee, Meng-Chan Hung, He-Yau Kang, W.L. Pearn(2012). A wind turbine evaluation model under a multi-criteria decision making environment. Energy Conversion and Management 64 289-300. 